\title{
Adaptive synchronization between chaotic dynamical systems of different order
}

\author{
Samuel Bowong ${ }^{1, *}$, Peter V. E. McClintock ${ }^{2}$ \\ 1 Laboratoire de Mathématiques Appliquées \\ Département de Mathématiques et Informatique, Faculté des Sciences \\ Université de Douala, B.P. 24157 Douala (Cameroun) \\ 2 Department of Physics, University of Lancaster, Lancaster LA1 4YB, UK \\ *Corresponding author \\ Tel. (237) 996-41-64, Fax. (237) 231-02-90 \\ Email: sbowong@uycdc.uninet.cm,p.v.e.mcclintock@lancaster.ac.uk \\ October 2, 2005 \\ This work discusses synchronization between two chaotic dynamical systems of different \\ order, using an adaptive control scheme. The problem is closely related to the synchronization \\ of strictly different chaotic systems. We show that the dynamical evolution of a fourth-order \\ system can be synchronized with canonical projections of a third-order system. In this sense, \\ it may be said that synchronization is achieved in reduced order, where by order we means the \\ number of first order differential equations. The mathematical stability analysis is derived from \\ the Lyapunov stability theory. Numerical simulations are presented to show the effectiveness \\ and feasibility of the proposed scheme.
}

Pacs numbers: 05.45.Xt, 05.45.Vx, 02.30.Yy 


\section{Introduction}

Coupled oscillatory systems are very common both in nature and in technology. Sometimes the coupling results in synchronization between the oscillations of the two systems - an effect first observed between a pair pendulum clocks by Huygens in 1665, while lying ill in bed. In 1990, Pecora and Carroll (PC) proposed a successful method [1] to synchronize two identical chaotic systems starting from different initial conditions. The idea of synchronization is to use the output of a master system to control a slave system so that the response of the latter follows the output of the master system asymptotically. Since then, chaos synchronization has been developed extensively as an important topic in its own right nonlinear science. It has been investigated by scientists working in fields as different as secure communications, optical, chemical, physical, and biological systems and neural networks. The synchronization of chaos has been reported in systems whose models are identical, similar, and with mismatched-parameters (see [2-6] for reviews). The synchronization of chaos can be induced even in strictly different oscillators [7-9] and systems of different order $[10,11]$. Nevertheless, given the diversity of phenomena that have been found in chaotic systems [12], there is not yet a clear and exact meaning of the synchronization in chaos. Many distinct kinds of synchronized systems are to be found in nature. Examples where we can expect to model synchronization between similar oscillators include nephron-nephron synchronization [13] and the mechanical systems [14]. On the other hand, nonidentical systems can be found in the synaptic communication where some neurons with different dynamic models can behave in a synchronous manner $[15,16]$. Another example is the synchronization that occurs between heart and lung, where one can observe that circulatory and respiratory systems synchronize [17], even through they are expected to be described by different models. Such facts add to the complexity of the meaning of the chaotic synchronization. In the nonlinear science, the definition of the synchronous behavior means that the trajectories of two or more dynamical systems evolve, in some sense, close to one and other along their trajectories.

An alternative approach to the understanding of synchronization between chaotic systems is to consider the design of the feedback structure: that is, the synthesis of the 
feedback control needed to synchronize chaotic systems. Efforts have been oriented in two directions, exploiting the properties of dynamical systems: their observability and their controllability. In the former case, the reconstruction of the drive system attractor from the response system is interpreted as an observer [18]. An interesting point about the observability of the synchronization systems is that differential geometry allows one to find an invariant space under vector fields where the attractor can be reconstructed (see Chapter 1 of [10]). On the other hand, the synchronization of chaotic systems has been also studied through the design of a controller trying to connect the departures of measured variables [7,19-21]. The synchronization is then understood as a stabilization problem and the controllability property is exploited. In other words, the aim is to compute the control input such that the difference between the trajectories of the slave system $y(t)$ remains close to the trajectories of the master system $x(t)$. That is, the point is to find the invariant space such that the origin of the synchronization error system $\|y(t)-x(t)\|=0$ can be stabilized. The observability and controllability of nonlinear systems are both included within the geometrical control theory $[22,23]$.

By the end of 90's, some efforts had been made to apply the controller approach to synchronize chaotic systems described by different models $[15,16,24-29]$. The underlying idea is to find a synchronization control signal, such that the existence of a synchronization manifold can be assured. In this manner, several synchronization phenomena were found [12], and different design techniques were exploited [24-29]. More recently, the synchronization of chaotic systems of different order was stated as a new problem [10]. The idea was to show that a second-order driven system can be synchronized with the projection on canonical planes of a third-order chaotic system. A nonlinear feedback interconnection was performed empirically such that synchronization was attained between the Duffing equation and the canonical-plane projection of the Chua's circuit. Of course, since the order of the response oscillator is less than the order of the master system, synchronization is only attained in reduced order. The following questions were formulated. Can reduced-order synchronization be attained by fourth-order and second-order systems? Can reduced-order synchronization be achieved by fourth-order and third-order systems? The results in [11] have shown evidence that synchronization of a fourth-order with a second- 
order system can be achieved. In this sense, the synchronization of the emitter-receiver system and Duffing equation is achieved in reduced order, i.e., the Duffing equation can be synchronized under feedback actions with the canonical projections of the emitter-receiver system.

In this paper, we discuss in detail chaotic synchronization between fourth-order and third order systems from the perspective of control theory. The master system that we consider is the Matsumoto-Chua-Kobayashi (MCK for short) circuit, while the slave system is Chua's circuit. It is known that active control is in general an effective method for bringing about synchronization between two different chaotic systems and this is the approach that we adopt in this work. Then, the stability of error signals between the drive and response systems is easily proved by adaptive linear control theory. In particular, the reduced-order synchronization is interpreted as a stabilization problem. The main idea is to design a control loop in the slave system so that the feedbacks steer the states of the synchronization error at the origin, i.e., all the states of the slave system track one part of the states of the master system.

The paper is presented as follows. In the next section, the reduced-order synchronization problem is stated. The synchronization strategy is then described in section 3. Numerical results are provided to illustrate the effectiveness and efficiency of the proposed adaptive synchronization methodology in section 4 . The text closes with concluding remarks in section 5 .

Throughout this paper, $\lambda(W)$ denotes an eigenvalue of $W, \lambda_{\max }(W)$ and $\lambda_{\min }(W)$ represent, respectively, the $\max \left[\lambda_{i}(W)\right]$ and the $\min \left[\lambda_{i}(W)\right], i=1, \ldots, n .|w|$ represents the absolute value of $w$, and $\|W\|$ represents the Euclidian norm when $W$ is a vector or the induced norm when $W$ is a matrix.

\section{Problem formulation}

The first system in which we are interested is the MCK circuit, which provided the first experimental observation of hyperchaos in a real physical system. By considering the equations reported in [30], the dynamics of the circuit can be written in non-dimensional 
form:

$$
\left\{\begin{array}{l}
\dot{x}_{1}=\gamma_{1}\left(f\left(x_{2}-x_{1}\right)-x_{3}\right) \\
\dot{x}_{2}=-\gamma_{2}\left[f\left(x_{2}-x_{1}\right)+x_{4}\right] \\
\dot{x}_{3}=\gamma_{3} x_{1}+\gamma_{4} x_{3} \\
\dot{x}_{4}=\gamma_{5} x_{2}
\end{array}\right.
$$

where the variables $x_{1}$ and $x_{2}$ represent the voltages across the two capacitors, while the variables $x_{3}$ and $x_{4}$ are the currents through the two conductors, and $f$ is the piecewise continuous function:

$$
f(x)=\gamma_{6} x+\frac{1}{2}\left(\gamma_{7}-\gamma_{6}\right)(|x+1|-|x-1|) .
$$

Let us consider the system's parameters (see [30]): $\gamma_{1}=2, \gamma_{2}=20, \gamma_{3}=1, \gamma_{4}=1$, $\gamma_{5}=1.5, \gamma_{6}=3$ and $\gamma_{7}=-0.2$. This circuit exhibits a typical hyperchaotic behavior (see Fig. 1), emphasized by computing the Lyapunov exponents (two of them are positive [30]). Initial conditions were arbitrarily located at the origin.

Our second system is Chua's circuit, which is a simple and interesting electronic system consisting of one inductor, two capacitors and one piecewise-linear nonlinear resistor. The mathematical model equations [31] for this circuit are

$$
\left\{\begin{array}{l}
\left.\dot{y}_{1}=\delta_{1}\left(y_{2}-y_{1}-g\left(y_{1}\right)\right)\right)+u_{1}, \\
\dot{y}_{2}=y_{1}-y_{2}+y_{3}+u_{2}, \\
\dot{y}_{3}=-\delta_{2} y_{2}+u_{3},
\end{array}\right.
$$

where $g\left(y_{1}\right)$ is a piecewise linear function represented by

$$
g\left(y_{1}\right)= \begin{cases}\gamma_{3} y_{1}+\delta_{4}-\delta_{3}, & y_{1} \geq 1 \\ \delta_{4} y_{1}, & \left|y_{1}\right| \leq 1 \\ \delta_{3} y_{1}-\delta_{4}+\delta_{3}, & y_{1} \leq 1\end{cases}
$$

In Eq. (2), variables $y_{1}$ and $y_{2}$ represent the voltages across the two capacitors, variable $y_{3}$ is the current through the inductor, and $u_{1}, u_{2}$ and $u_{3}$ stand for the synchronization forces (i.e., the couplings between the master and slave systems such that they are synchronous). It is known that for system parameters $\delta_{1}=10, \delta_{2}=14.87, \delta_{3}=-0.68$ and $\delta_{1}=-1.27$, a typical chaotic attractor (a double scroll) can be found in dynamical system (2). The 
chaotic attractor obtained is displayed in Fig. 2 for $u_{1}=u_{2}=u_{3}=0$ (uncontrolled evolution). Initial conditions were arbitrarily located at the point $\left(y_{1}(0), y_{2}(0), y_{3}(0)\right)=$ $(0.1,0,0,0)$.

There are obviously differences between the attractors of the MCK system and of Chua's circuit, i.e., the MCK system and Chua's circuit are synchronized in neither phase nor frequency (see phase portraits in Fig. 2 and projections in Fig. 1). Moreover, there is no synchronization in any sense (see Refs. [12,19] for definitions of different kinds of synchronization). Systems (1) and (2) have been widely used to study chaos synchronization when drive and response system are of the same order.

Following the ideas of $[10,11]$, we can now state the synchronization problem as follows: To find scalar driving forces $u_{1}, u_{2}$ and $u_{3}$ such that the system (2) is synchronous with canonical projections (reduced-order synchronization) of the MCK system (1) for $t>0$ and any initial conditions $\left(x_{1}(0), x_{2}(0), x_{3}(0), x_{4}(0)\right)$ and $\left(y_{1}(0), y_{2}(0), y_{3}(0)\right)$.

In the next section, the detailed design procedure of the feedback control laws $u_{1}, u_{2}$ and $u_{3}$ is described with detailed explanations.

\section{Adaptive chaos synchronization algorithm}

In this section, an adaptive control scheme will be developed to attain the synchronization objective stated above. To this end, let us define the synchronization state errors between the response system (2) that is to be controlled and the controlling system (1) as $e_{1}=y_{1}-x_{1}, e_{2}=y_{2}-x_{2}$ and $e_{3}=y_{3}-x_{3}$. Subtracting the second system (2) from the first system (1) which includes the control signals, we obtain

$$
\left\{\begin{array}{l}
\dot{e}_{1}=\delta_{1}\left(e_{2}-e_{1}\right)+\zeta\left(e_{1}, x_{1}\right)+\phi_{1}(x)+u_{1}, \\
\dot{e}_{2}=e_{1}-e_{2}+e_{3}+\phi_{2}(x)+u_{2}, \\
\dot{e}_{4}=-\delta_{2} e_{2}+\phi_{3}(x)+u_{3},
\end{array}\right.
$$


where $x=\left(x_{1}, x_{2}, x_{3}, x_{4}\right)^{T}$ and

$$
\begin{aligned}
\zeta\left(e_{1}, x_{1}\right) & =-\delta_{1} g\left(e_{1}+x_{1}\right), \\
\phi_{1}(x) & =\delta_{1} x_{2}-\delta_{1} x_{1}-\gamma_{1} f\left(x_{2}-x_{1}\right)+\gamma_{1} x_{3}, \\
\phi_{2}(x) & =x_{1}-x_{2}+x_{3}+\gamma_{2}\left(f\left(x_{2}-x_{1}\right)+x_{4}\right), \\
\phi_{3}(x) & =-\delta_{2} x_{2}-\gamma_{3} x_{4}-\gamma_{4} x_{3},
\end{aligned}
$$

can be interpreted as the action of disturbing forces on the linear part of system (3). The synchronization error to be controlled is thus a nonlinear system with control inputs $u_{1}$, $u_{2}$ and $u_{3}$ as a function of the synchronization error states $e_{1}, e_{2}$ and $e_{3}$. Eq. (3) may be rewritten in the form of a matrix equation:

$$
\dot{e}=A e+u+B \zeta\left(e_{1}, x_{1}\right)+\phi(x),
$$

where $e=\left(e_{1}, e_{2}, e_{3}\right)^{T}, \phi(x)=\left(\phi_{1}(x), \phi_{2}(x), \phi_{3}(x)\right)^{T}, u=\left(u_{1}, u_{2}, u_{3}\right)^{T}$,

$$
A=\left[\begin{array}{ccc}
-\delta_{1} & \delta_{1} & 0 \\
1 & -1 & 1 \\
0 & -\delta_{2} & 0
\end{array}\right] \quad \text { and } \quad B=\left[\begin{array}{l}
1 \\
0 \\
0
\end{array}\right]
$$

The synchronization problem may thus be formulated as follows: To find the driving force $u \in \mathbb{R}^{3}$ such that the discrepancy error $e(t)$ which starts at $e_{0}=e(0)$ at $t_{0}=0$ tends asymptotically to zero as $t \rightarrow \infty$. Note that the above goal implies that if $e(t) \rightarrow 0$ as $t \rightarrow \infty$, then $x_{1}(t) \rightarrow y_{1}(t), x_{2}(t) \rightarrow y_{2}(t)$ and $x_{3}(t) \rightarrow y_{3}(t)$ for all $t \geq 0$. In order to do so, some necessary assumptions must be made as follows:

Assumption 1: The nonlinear function $\zeta\left(e_{1}, x_{1}\right)$ is globally Lipschitz, i.e., there exists a positive constant $k$ such that

$$
\left\|\zeta\left(e_{1}, x_{1}\right)-\zeta\left(0, x_{1}\right)\right\| \leq k\|e\|, \quad \forall x_{1} \in \mathbb{R} .
$$

Assumption 2: For the uncertain nonlinear function $\phi(x)$, there exists a positive constant large enough that

$$
\|\phi(x)\|<\phi_{m}<\infty
$$

where $\phi_{m}>0$ is an unknown and sufficiently large constant.

Assumption 3: There exists a constant matrix

$$
K=\left[\begin{array}{ccc}
k_{1} & 0 & 0 \\
0 & k_{2} & 0 \\
0 & 0 & k_{3}
\end{array}\right]
$$


and two positive definite matrices $P=P^{T}$ and $Q=Q^{T}$ such that the following algebraic equations hold:

$$
(A-K)^{T} P+P(A-K)=-Q .
$$

Some comments regarding the above assumptions are in order. Concerning Assumption 1, the Lipschitz constant $k$ is often required to be known for the control design purpose. In fact, it is often difficult to obtain a precise value of $k$ in some practical systems, hence the Lipschitz constant is often selected to be larger, which will induce the control gain to be higher, and the obtained results would be conservative. Assumption 2 is reasonable for the boundness of the chaotic attractor in state space and the interaction of all trajectories inside the attractor. Note also that the constant $\phi_{m}$ is only introduced to prove Theorem 1 later and does not appear in our proposed adaptive feedback controllers: we can suppose that $\phi_{m}$ is a constant large enough (i.e., $\phi_{m} \rightarrow \infty$ ) such that Assumption 2 is always satisfied.

If the upper bound of the uncertainty $\phi(x)$ is known, i.e. $\phi_{m}$ is known a priori, then a robust control law $u(t)$ in (4) can be designed to overcome the effect of unknown parametric perturbations, and further to achieve synchronization. The main problem is that $\phi_{m}$ is unknown. Therefore, an adaptive robust control law is needed. It may be designed as follows:

$$
u=-\frac{1}{2} \alpha e,
$$

where $\alpha$ is the estimated feedback gain which is updated on-line by the following adaptation law:

$$
\dot{\alpha}=\gamma \lambda_{\min }(P)\|e\|^{2} .
$$

in which $\gamma$ is a suitable positive constant.

Substitution of the adaptive feedback controllers (8) into (4) leads to

$$
\dot{e}=A e+B \zeta\left(e_{1}, x_{1}\right)+\phi(x)-\frac{1}{2} \alpha e .
$$

We can now give our main result.

Theorem 1 : If Assumptions 1-3 are satisfied, then the slave Chua circuit (2) associated with the adaptive control laws (8)-(9) globally synchronizes the drive MKC system (1) in reduced-order, i.e, $x_{1}(t) \rightarrow y_{1}(t), x_{2}(t) \rightarrow y_{2}(t)$ and $x_{3}(t) \rightarrow y_{3}(t)$ as $t \rightarrow \infty$. 
Proof: By adding and subtracting the term Ke, the closed-loop system (10) may be rewritten as

$$
\dot{e}=(A-K) e+K e+B \zeta\left(e_{1}, x_{1}\right)+\phi(x)-\frac{1}{2} \alpha e .
$$

Consider the following Lyapunov function candidate:

$$
V(t)=e^{T} P e+\frac{1}{2 \gamma}(\bar{\alpha}-\alpha)^{2}
$$

where $P=P^{T}$ is the positive definite solution of Eq. (7) and $\bar{\alpha}$ is given by

$$
\bar{\alpha}=\frac{1}{\lambda_{\min }(P)}\left[\frac{k^{2}}{\varepsilon}+\frac{\left(\phi_{m} \lambda_{\max }(P)\right)^{2}}{\beta}\right],
$$

with $\varepsilon$ and $\beta$ two suitable positive constants. It can easily verify that $V(t)$ is a nonnegative function and that it is radically unbounded, i.e., $V(t) \rightarrow+\infty$ as $e(t)$ and $(\bar{\alpha}-\alpha)$ $\rightarrow+\infty$. The time-derivative of $V(t)$ along the trajectories of system (11) satisfies

$$
\begin{aligned}
\dot{V}(t) & =e^{T}\left[(A-K)^{T} P+P(A-K)\right] e+2 e^{T} P K e+2 e^{T} P B \zeta\left(e_{1}, x_{1}\right) \\
& +2 e^{T} P \phi(x)-\alpha e^{T} P e-\frac{1}{\gamma}(\bar{\alpha}-\alpha) \dot{\alpha} \\
& \leq-e^{T}(Q-P K) e+2\|P e\| k\|e\|+2 \phi_{m}\|P\|\|e\|-\alpha\|P e\|^{2}-\frac{1}{\gamma}(\bar{\alpha}-\alpha) \dot{\alpha} .
\end{aligned}
$$

Notice that

$$
2\|P e\| k\|e\| \leq \frac{k^{2}}{\varepsilon}\|P e\|^{2}+\varepsilon\|e\|^{2},
$$

and

$$
2 \phi_{m}\|P\|\|e\| \leq \frac{\left(\phi_{m} \lambda_{\max }(P)\right)^{2}}{\beta}\|e\|^{2}+\beta
$$

Then, we get

$$
\begin{aligned}
\dot{V}(t) & \leq-\left[\lambda_{\min }(Q)-\lambda_{\max }(P K)-\varepsilon\right]\|e\|^{2}+\left[\frac{k^{2}}{\varepsilon}+\frac{\left(\phi_{m} \lambda_{\max }(P)\right)^{2}}{\beta}\right]\|e\|^{2} \\
& +\beta-\alpha \lambda_{\min }(P)\|e\|^{2}-\frac{1}{\gamma}(\bar{\alpha}-\alpha) \dot{\alpha}
\end{aligned}
$$

If we choose $\bar{\alpha}$ as in Eq. (13), then (17) will be

$$
\dot{V}(t) \leq-\left[\lambda_{\min }(Q)-\lambda_{\max }(P K)-\varepsilon\right]\|e\|^{2}+\beta+(\bar{\alpha}-\alpha)\left(\lambda_{\min }(P)\|e\|^{2}-\frac{\dot{\alpha}}{\gamma}\right) .
$$

Thus, by applying the adaptive law (9), one obtains

$$
\dot{V}(t) \leq-\left[\lambda_{\min }(Q)-\lambda_{\max }(P K)-\varepsilon\right]\|e\|^{2}+\beta .
$$


Let us define $D=\lambda_{\min }(Q)-\lambda_{\max }(P K)-\varepsilon$. Note that the free parameter $\varepsilon>0$ can be selected to be small enough to let $D>0$. From Eq. (19), it follows that if

$$
\|e\|>\sqrt{\frac{\beta}{D}}
$$

then, $\dot{V}(t)<0$, so that $V(t)$ decreases, which implies that $\|e\|$ decreases as well (see Eq. (12)). It then follows from the standard invariance argument that asymptotically, the error satisfies the bound

$$
\|e\| \leq c
$$

where $c \geq \sqrt{\frac{\beta}{D}}$, (see e.g. [32], page 231). From (20), it clearly appears that the asymptotic error depends linearly on the free parameter $\beta$. Hence, if this parameter is small, the resulting error will be small as well. The dependence of the error on $\varepsilon$ deserves special attention. Note that $D=\lambda_{\min }(Q)-\lambda_{\max }(P K)-\varepsilon$. Hence as $\varepsilon$ decreases, $D$ will increase, which decreases the asymptotic error bound. This argument shows that with the proposed method, $\varepsilon$ and $\beta$ should be made as small as possible. This implies that the Chua's system (2) associated with the adaptive laws (8), (9) can globally synchronize the drive MCK system $(1)$ in reduced-order, i.e., $x_{1}(t) \rightarrow y_{1}(t), x_{2}(t) \rightarrow y_{2}(t)$ and $x_{3}(t) \rightarrow y_{3}(t)$ as $t \rightarrow \infty$. This achieves the proof.

From Eq. (13), not only the unknown Lipschitz constant $k$ but also the unknown bound $\phi_{m}$ can be injected into the constant $\bar{\alpha}$, which can be adaptively adjusted by the adaptation law (9). Further, the global stability of the error dynamics (11) can be ensured if the constant $\varepsilon$ is sufficiently small.

\section{$4 \quad$ Numerical studies}

This section presents numerical results to verify the effectiveness of the proposed adaptive synchronization scheme. We first select $K=I$ where $I$ is the identity matrix of dimension 3 so that the the eigenvalues of matrix $A-K$ are -11.8886 and $-1.0557 \pm$ 3.6951i. The following symmetric and positive-definite matrices

$$
P=\left[\begin{array}{ccc}
0.1478 & 0.6554 & 0.0156 \\
0.6554 & 6.8845 & -0.4381 \\
0.0156 & -0.4381 & 0.5619
\end{array}\right] \quad \text { and } \quad Q=\left[\begin{array}{ccc}
2 & 0 & 0 \\
0 & 2 & 0 \\
0 & 0 & 2
\end{array}\right]
$$


are selected to satisfy Eq. (7). In this case $\lambda_{\min }(P)=0.0774$ and $\lambda_{\max }(P)=6.9771$.

Without lost of generality, one can consider that parameters and initial conditions of the master and slave systems have the same values as in Figs. 1 and 2.

Figure 3 shows the state trajectories of the MCK system (solid line) and of the Chua's circuit (dashed line). The controller was applied at $t=50$. On can see that when $u=0$, the trajectories diverge from each other. However, as soon as the adaptive controllers are applied, the states $x_{1}$ and $y_{1}, x_{2}$ and $y_{2}$ and $x_{3}$ and $y_{3}$ evolve together in an almost synchronous way. This implies that the response MCK system (1) globally synchronizes with the driving Chua's circuit (2) in spite of the fact that both master and slave systems have different models and orders. Note that the synchronization of systems (1) and (2) is attained in phase and practical sense. Under these conditions, one can expect that the double scroll of Chua's circuit must become the hyperchaotic attractors of the MCK system. Figure 4 shows the attractors of Chua's circuit under the action of the adaptive feedback: under the control actions of (8), (9) they have become exact images of the hyperchaotic attractors of Fig. 1, the attractors of the MCK system.

In order to provide additional evidence of the effectiveness and efficiency of the proposed adaptive synchronization scheme, we show in Fig. 5 the power spectra (PSDs) of $x_{1}, x_{2}, x_{3}, y_{1}, y_{2}$ and $y_{3}$ under feedback actions. The PSD has been normalized by the maximum amplitude peak in each case. Such a picture shows that, at least, frequency and phase synchronization is attained by the adaptive feedback control laws (8), (9). PSD is an important measure of synchronization. Although, PSD is not sufficient to conclude that synchronization exists, they provide good evidence [32]. Note that spectra in Chua's equations under control action are similar to than the MCK system $\left(y_{1}, y_{2}, y_{3}\right)$ projection.

\section{Conclusion}

In this paper, we have presented a robust control scheme for synchronizing fourth-order and third-order chaotic systems. In particular, we have demonstrated the matching of the Chua attractor with the projection on canonical planes of the MCK system. The feedback couplings were designed with a simple algorithm based on an adaptive control strategy of chaos suppression. The main advantage of the proposed adaptive synchronization scheme 
is that the bound on uncertainties is unknown. Numerical simulations are given to validate the efficiency and effectiveness of the proposed synchronization approach. 


\section{References}

[1] Caroll, L. M. and Pecora, T. L., Phys. Rev. Lett. 38, 453 (1991).

[2] G. Chen, X. Dong, From chaos to order: methodologies, perspectives and applications (Singapore: World Scientific, 1998).

[3] M. Lakshamanam, K. Murali, Chaos in Nonlinear Oscillators: Controlling and Synchronization (Singapore: World Scientific, 1996).

[4] Pikovsky, A., M. Rosenblum and J. Kurths, Synchronization: a universal concept in nonlinear sciences ( New York: Cambridge University Press, 2001).

[5] B. R. Andrievskii and A. L. Fradkov, Control of chaos: methods and applications I. Methods, Autom. Rem. Control 64, 3 (2003); B. R. Andrievskii and A. L. Fradkov, Control of chaos: methods and applications I. Applications, Autom. Rem. Control 65, 3 (2004).

[6] S. Bocaletti, J. Kurths, G. Osipov, D. Valladares, and C. Zhou, Phys. Rep. 366, 1 (2002).

[7] G. Xiaofeng and C. H. Lai, Chaos Solitons \& Fractals 11, 1231 (2000).

[8] R. Femat and J. Alvarez-Ramirez, Phys. Lett. A 236, 307 (1997).

[9] S. Bowong and F. M. Moukam, Phys. Scripta 68, 636 (2003).

[10] R. Femat and G. Solis-Perales, Phys. Rev. E 65, 036226 (2002).

[11] S. Bowong, Phys. Lett. A 326, 102 (2004).

[12] R. Femat and G. Solis-Perales, Phys. Lett. A 262, 50 (1999).

[13] N. H. Holstein-Rathlou, K. P. Yip, O. V. Sosnovtseva and E. Mosekilde, Chaos 11, 417 (2001).

[14] H. Nijmeijer and A. Rodríguez-Angeles, Synchronization of mechanical systems (World Scientific Series on Nonlinear Science, Series A - Vol. 46, 2003). 
[15] D. Terman, N. Kopell and A. Bose, Physica D 117, 241 (1998).

[16] M. Bezhenov, R. Huerta, M. I. Rabonovich and T. Sejnowski, Physica D 116, 392 (1998).

[17] A. Stefanovska, H. Haken, P. V. E. McClintock, M. Hozic, F. Bajrovic and S. Ribaric, Phys. Rev. Lett. 85, 4831 (2000).

[18] H. Nijmeijer and M. Y. Mareels, IEEE Trans. Circuits Syst. I 44, 882 (1997).

[19] R. Brown, and L. Kocarev, Chaos 10, 344 (2000).

[20] R. Femat, J. Alvarez-Ramirez and G. Fernandez-Anaya, Physica D 139, 231 (2002).

[21] S. Bowong, F. M. Moukam Kakmeni and C. Tchawoua, Phys. Rev. E 70, 066217 (2004).

[22] A. Isidori, Nonlinear control systems (3th Ed., Springer-Verlag, London, 1995).

[23] H. Nijmeijer and A. Van der Schaft, Nonlinear control dynamical systems (SpringerVerlag, 1990).

[24] L. Kocarev and U. Parlitz, Phys. Rev. Lett. 76, 1816 (1996).

[25] T. Yang and H. H. Shao, Phys. Rev. E 65, 046210 (2002).

[26] S. Bowong and F. M. Moukam Kakmeni, Phys. Scripta 68, 326 (2003).

[27] M. T. Yassen, Chaos, Solitons \& Fractals 15, 271 (2003).

[28] H. Fotsin, S. Bowong and J. Daafouz, Chaos, Solitons \& Fractals 26, 215 (2005).

[29] U. Parlitz, L. Junge and L. Kocarev, Int. J. Bifur. Chaos 9, 2305 (1999).

[30] T. Matsumoto, L. O. Chua and K. Kobayashi, IEEE Trans. Circ. Syst. -I 33, 1143 (1986).

[31] R. N. Madan, Chua's circuit: a paradigm for chaos (In World Scientific series on nonlinear science Series B, special theme issues and proceedings, vol. 1. Singapore: World Scientific, 1993). 
[32] H. K. Khalil, Nonlinear systems (New York: Macmillan, 1992). 


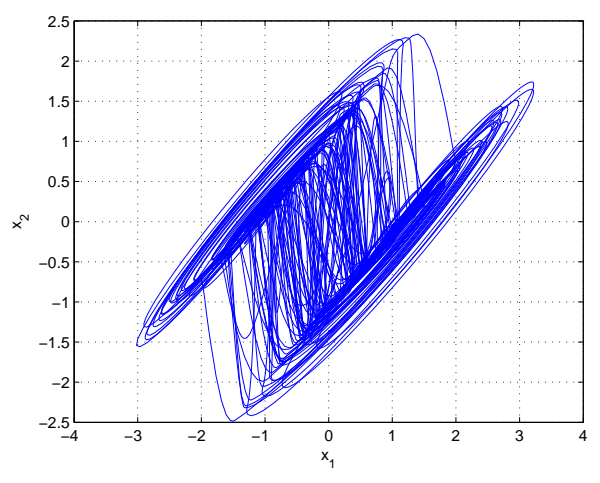

(a)

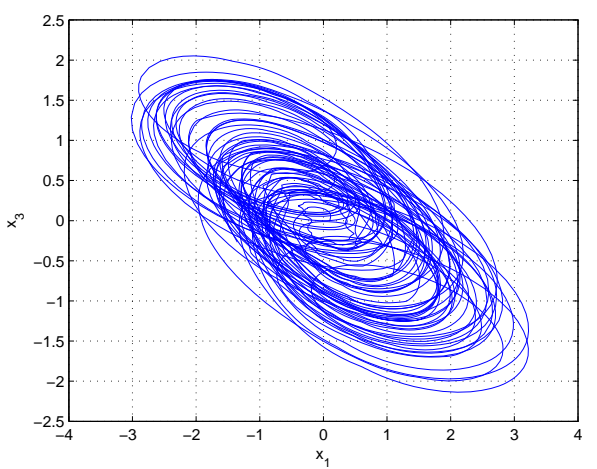

(b)

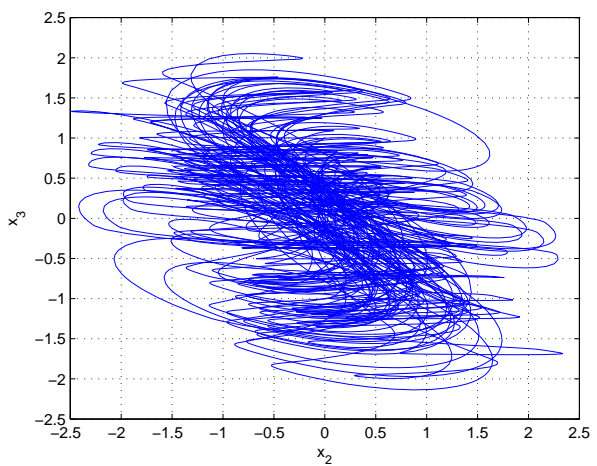

(c)

Figure 1: Hyperchaotic attractors of the MCK system. The parameters are provided in the text. 


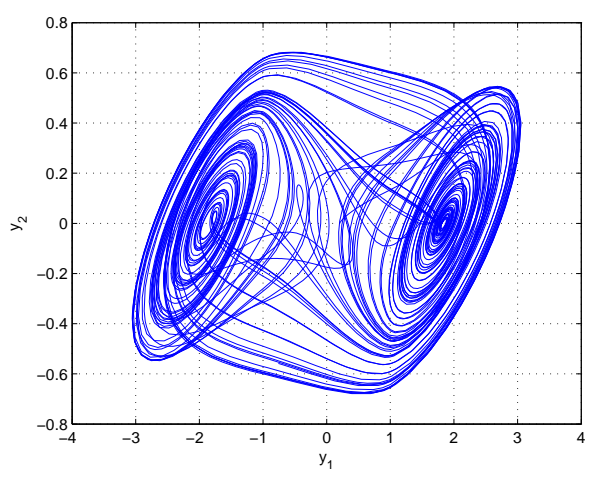

(a)

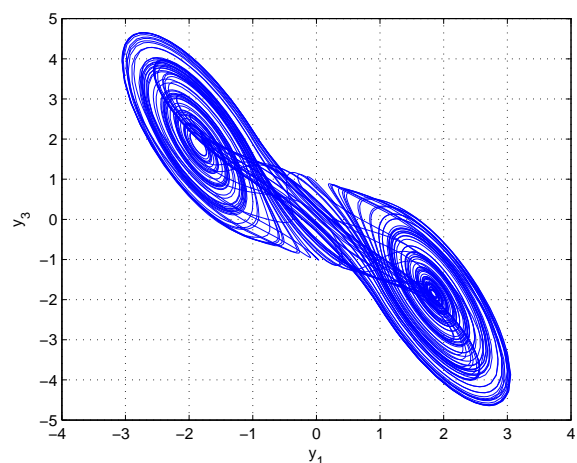

(b)

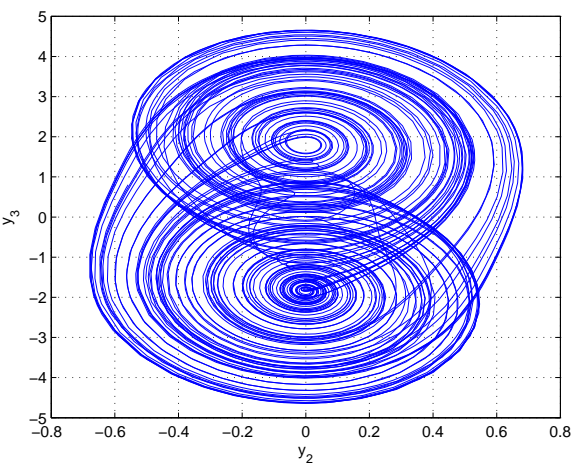

(c)

Figure 2: Double scroll attractors of the Chua's circuit system without feedback couplings. The parameters are provided in the text.
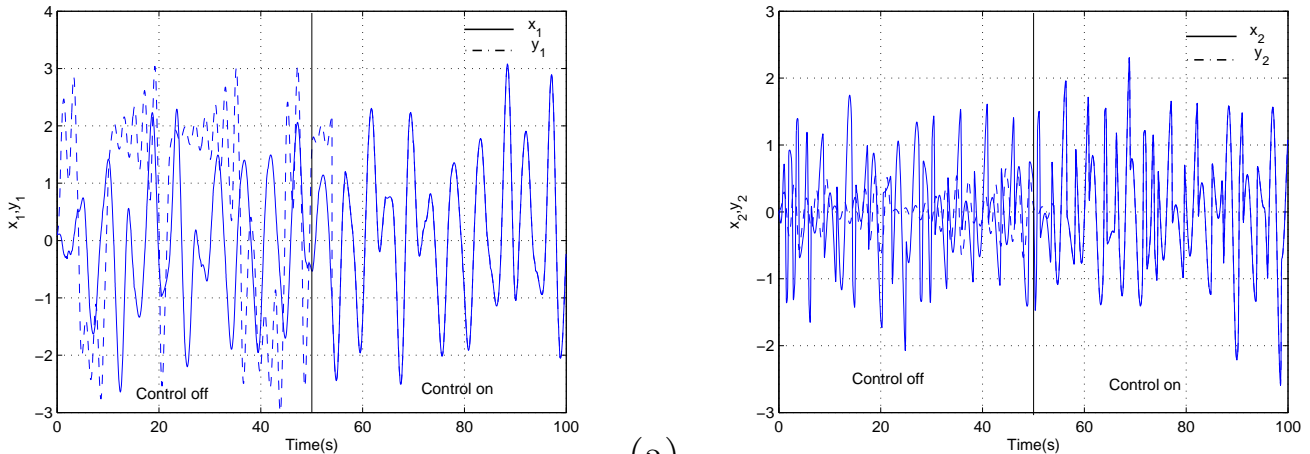

(a)

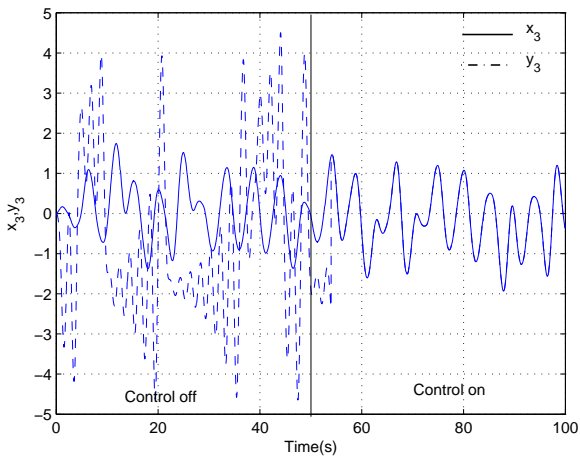

(c)

Figure 3: State trajectories of $x_{1}, x_{2}, x_{3}$ (solid line) and $y_{1}, y_{2}, y_{3}$ (dashed line), respectively. 


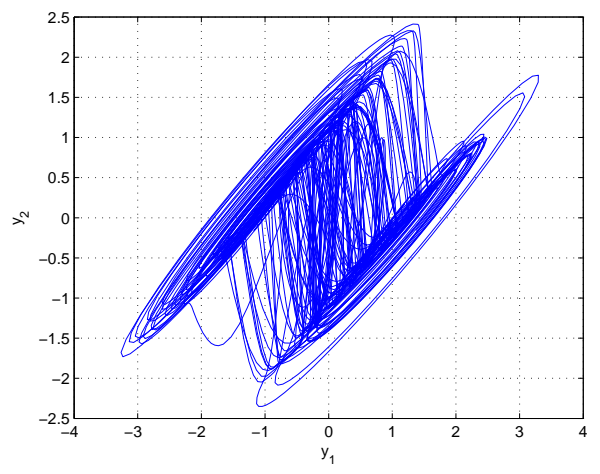

(a)

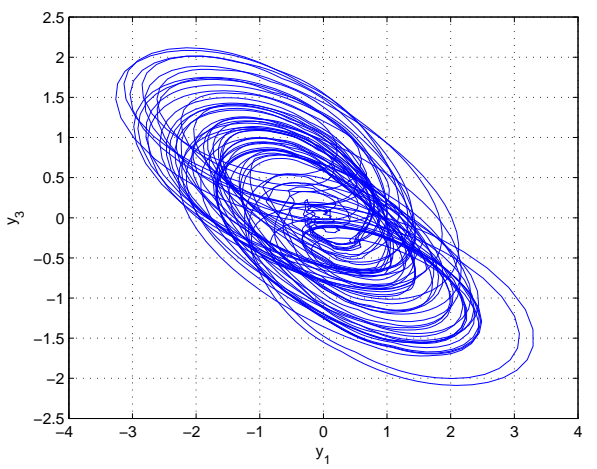

(b)

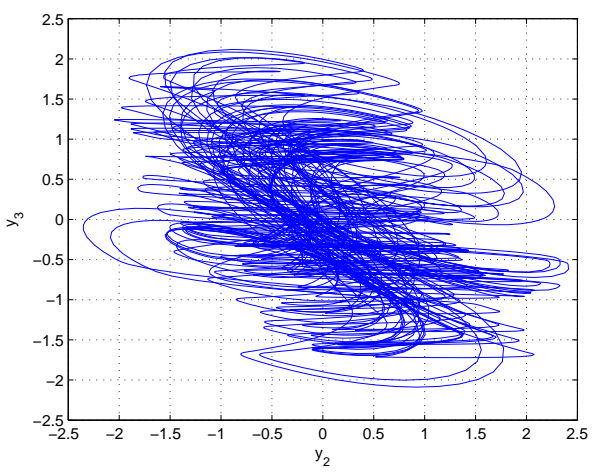

(c)

Figure 4: Phase portraits of the Chua's circuit under feedback actions. 


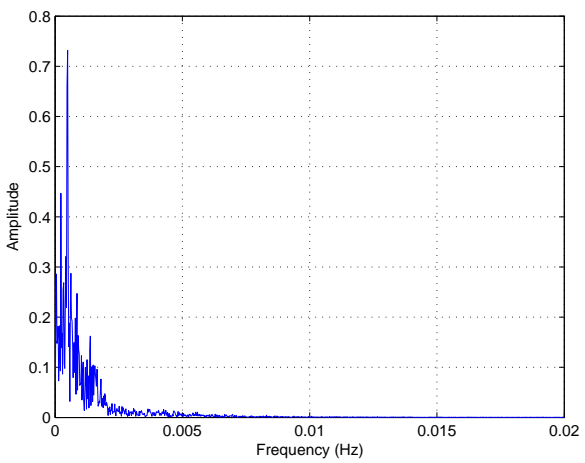

(a)

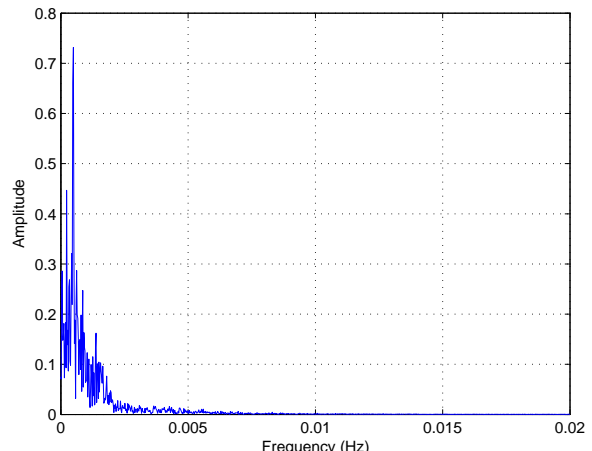

(d)

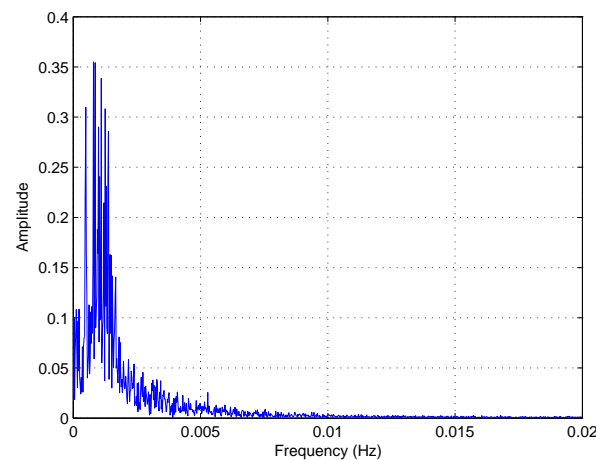

(b)

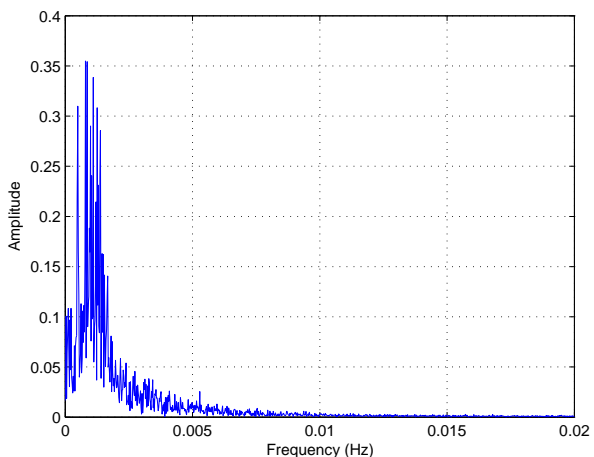

(e)

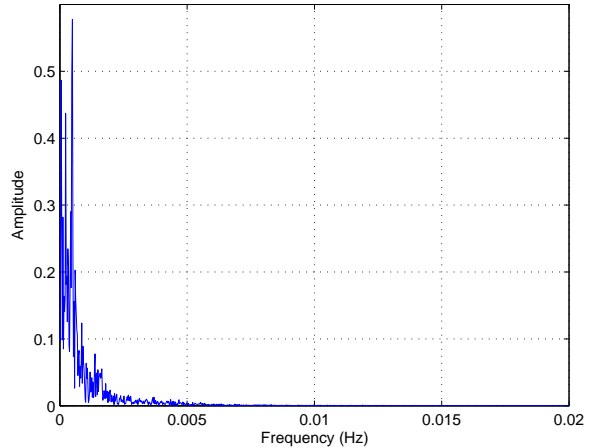

(c)

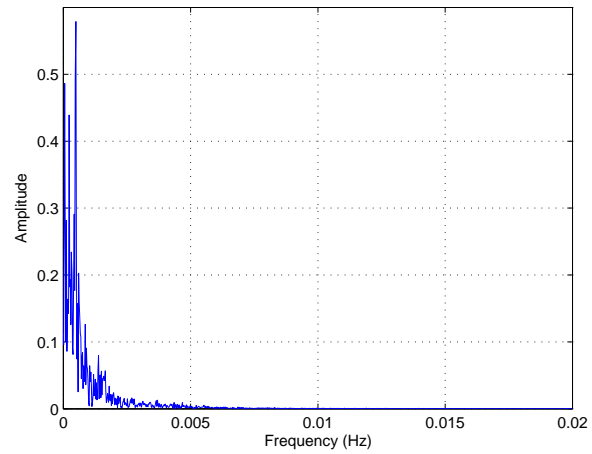

(f)

Figure 5: Power spectrum of (a) $x_{1}$; (b) $x_{2}$; (c) $x_{3}$ and (d) $y_{1}$; (e) $y_{2}$; (f) $y_{3}$ under control actions. 